\title{
Aspectos ecológicos de Heteropteras Aquáticos e Semi-aquáticos no Leste
}

\section{Maranhense}

\author{
Ecological aspects of Aquatic and Semi-Aquatic Heteroptera in East Maranhense \\ Aspectos ecológicos de Las Heteropteras Acuáticas y Semiacuáticas en el Este de Maranhense
}

Recebido: 19/11/2021 | Revisado: 27/11/2021 | Aceito: 02/12/2021 | Publicado: 13/12/2021

Francisca Barbara e Silva Barros

ORCID: https://orcid.org/0000-0002-5734-0431 Universidade Estadual do Maranhão, Brasil E-mail: barbarabarros1234@gmail.com

Carlos Augusto Silva de Azevêdo

ORCID: https://orcid.org/0000-0002-0503-3843

Universidade Estadual do Maranhão, Brasil E-mail: casazevedo08@gmail.com

Cleilton Lima Franco

ORCID: https://orcid.org/0000-0003-1827-773X Universidade Federal Rural do Rio de Janeiro, Brasil E-mail: cleiltonubc@hotmail.com

\begin{abstract}
Resumo
O objetivo foi descrever aspectos ecológicos da subordem Heteroptera e associar a alguns fatores abióticos e características ambientais. As coletas foram realizadas em seis igarapés, sendo realizado em cada local um transecto de $50 \mathrm{~m}$, dividido em cinco pontos de $10 \mathrm{~m}$, nos quais os espécimes foram coletados com auxílio de uma rede entomológica aquática rede em D (rapiché), peneira e catação manual em substratos como folhas, troncos, macrófitas, raízes, entre outros. Antes das coletas dos espécimes, obteve-se dados da vegetação ripária, tipos de leito, áreas de remanso e correnteza, temperatura, $\mathrm{pH}$, condutividade elétrica, largura, profundidade, velocidade, vazão e cobertura de dossel. Os dados da riqueza dos táxons por igarapé foram estimados pelo teste de riqueza não-paramétricos de Jackknife; para análise dos paramentos físico e ambientais foram utilizados as Análise de Coordenadas Principais, Análise de Redundância; sendo que a associação entre as amostras foi verificada pela matriz de similaridade de BrayCurtis, após os dados serem transformados por $\log (\mathrm{x}+1)$. Foram coletados 269 heteropteros aquáticos, identificados em duas infraordens Gerromorpha e Nepomorpha com sete famílias e 17 gêneros. Os Locais mais heterogêneos entre si apresentam uma maior riqueza de gêneros, apesar da morfologia e especificidade de habitat possa influenciar sua preferência. O riacho Jatobá se apresentou a maior riqueza, juntamente com as análises o riacho teve uma melhor correlação a maioria dos gêneros enquanto que o Baixa Grande teve maior relação com a maioria das variáveis ambientes e apenas relação com o gênero Pelocoris, e o gênero que melhor se correlacionou com o substrato foi Brachymetra, o conhecimento da riqueza e da ecologia determina a sensibilidade de ecossistemas e os gêneros que nele vivem se faz necessário para conservação.
\end{abstract}

Palavras-chave: Diversidade; Gerromorpha; Insetos; Nepomorpha.

\begin{abstract}
This article has the purpose of describing suborder Heteroptera's ecological aspects and associating them with some abiotic factors and environmental particulars. The gathering was made in six rivers, with a 50m transect divided among five $10 \mathrm{~m}$ spots, where species were ghathered using an aquatic entomological D-net (rapiché), a sieve and manual scavenging on substrates incluinding leaves, trunks, macrophytes, roots, and others. Before the species gathering, it was obtained riparian vegetation data, bed types data, backwater and river áreas data, temperature data, $\mathrm{pH}$ data, electrical conductivity data, width data, depth data, velocity data, flow and canopy cover data. Besides, taxon richness data in the river were estimated by the non-parametric Jackknife richness test; for the physical and environmental parameters analysis, were used the Principal Coordinate Analysis and Redundancy Analysis; and the association between specimen was verified by the Bray-Curtis similarity matrix, after the data were transformed by $\log (x+1)$. Were gathered 269 aquatic heteropterans, identified in two infraorders Gerromorpha and Nepomorpha with seven families and 17 genus. The most heterogeneous spots showed a higher richness of genus, although morphology and habitat specificity might influence their preference. The Jatobá river showed the greatest richness, with the analyses. This river had a better correlation with majority of genus, while the Baixa Grande Creek had a greater relation with most of the environmental variables and only a relation with the genus Pelocoris, and the genus which correlated with the substrate in a better way was Brachymetra. Richness and Ecology's knowledging ascertains the ecosystems' sensitivity and the living genus is required for conservation.
\end{abstract}

Keywords: Diversity; Gerromorpha; Insects; Nepomorpha. 


\begin{abstract}
Resumen
En ese artículo, se describen los aspectos ecológicos del suborden Heteroptera y asociarlo con algunos factores físicoquímicos y ambientales. Las recolecciones se realizaron en seis arroyos, realizándose un transecto de $50 \mathrm{~m}$ en cada sitio, dividido en cinco puntos de $10 \mathrm{~m}$, en los que los ejemplares fueron recolectados con la ayuda de una red entomológica acuática en D (rapiche), cedazo y recolección manual. en sustratos como hojas, troncos, macrófitas, raíces, entre otros. Antes de la recolección de la muestra, se obtuvieron datos sobre vegetación ribereña, tipos de lecho, áreas de remanso y corriente, temperatura, $\mathrm{pH}$, conductividad eléctrica, ancho, profundidad, velocidad, flujo y cobertura del dosel. Los datos de riqueza de los taxones por corriente se estimaron mediante la prueba de riqueza no paramétrica Jackknife; Para el análisis de los parámetros físicos y ambientales, el Análisis de Coordenadas Principales, Análisis de Redundancia; la asociación entre las muestras se verificó mediante la matriz de similitud de Bray-Curtis, los datos transformados por $\log (x+1)$. Se recolectaron un total de 269 heteroptera acuáticos, identificados en dos infraórdenes Gerromorpha y Nepomorpha con siete familias y 17 géneros. Los sitios que son más heterogéneos entre sí tienen una mayor riqueza de especies, aunque la morfología y la especificidad del hábitat pueden influir en su preferencia. El arroyo Jatobá tuvo la mayor riqueza, junto a las análisis, el arroyo tuvo una mejor correlación con la mayoría de las especies mientras que el Baixa Grande tuvo una relación más alta con la mayoría de las variables ambientales y solo una relación con la especie Pelocoris, y el género que mejor se correlacionó con la el sustrato fue Brachymetra, el conocimiento de la riqueza y la ecología determina la sensibilidad de los ecosistemas y las especies que habitan en él es necesario para su conservación.
\end{abstract}

Palabras clave: Diversidad; Gerromorpha; Insectos; Nepomorpha.

\title{
1. Introdução
}

A ordem Hemiptera é constituída como a maior e mais diversa entre os insetos hemimetabólicos, com cerca de 89.000 espécies distribuídas mundialmente. A ordem está dividida nas subordens Auchenorrhyncha, Sternorrhyncha, Coleorrhyncha e Heteroptera, em que todos os táxons aquáticos neotropicais estão na subordem Heteroptera. Além disso, está dividida em sete infraordens: Enicocephalomorpha, Dipsocoromorpha, Gerromorpha, Nepomorpha, Leptopodomorpha, Cimicomorpha e Pentatomomorpha (Štys e Kerzhner, 1975).

Os membros das infraordens Gerromorpha, Nepomorpha e Leptopodomorpha estão associados a ambientes dulcícolas, possuindo algumas taxas tolerantes ou restritas à água salgada ou secundariamente terrestre (Schuh e Slater, 1995); as três infraordens conjuntas incluem cerca de 4.800 espécies, mas pouco diversificadas se comparadas às terrestres. A Leptopodomorpha é a menos rica em espécies, com cerca de 380 espécies. Já a Gerromorpha com cerca de 2.100 e Nepomorpha contendo mais de 2.300 pelo mundo (Polhemus e Polhemus, 2008).

Já na região neotropical, apresentam-se 18 Famílias e 1.289 espécies, sendo essa, a mais rica do mundo em termos de espécies descritas. A Leptopodomorpha com 42 espécies, a Gerromorpha com cerca 515 e a Nepomorpha com 723 (Polhemus e Polhemus, 2008), No Brasil, Moreira et al., (2011) publicaram um catálogo listando as espécies ocorrentes de ambas as infraordens, em que registraram 479 espécies ao todo, sendo 187 (39\%) de Gerromorpha e 292 (61\%) de Nepomorpha.

Os heterópteros podem viver em diversos ambientes aquáticos, como rios, igarapés, lagos de altitude ou salinos, nascentes, poças de água, em ambientes marinhos, como planctônicos ou associados a plantas (ex: bromeliáceas epífitas), troncos, raízes e folhas submersas (Bachmann, 1998; Hamada e Ferreira-Keppler, 2012). Graças a alta capacidade de dispersão, algumas espécies se tornam pioneiras na colonização de corpos d’água (Bachmann, 1998) ou indicadores de qualidade ambiental.

Nos ambientes aquáticos, a presença de mata ciliar, de abertura do dossel, de integridade de habitat, de intensa cobertura vegetal, de altitude e vazão, de velocidade da correnteza em sistemas lóticos, de pH, de condutividade elétrica, de disponibilidade de alimento, de temperatura, de oxigênio dissolvido e de tipos de substratos, são as principais variáveis relacionadas à fauna de heterópteros aquáticos e semi-aquáticos (Merritt e Cummins, 1996; Kikuchi e Uieda, 1998; Goulart et al., 2002; Couceiro et al., 2010; Dias-Silva et al., 2010; Ilie e Olosutean, 2013; Peiró et al., 2013) em que as modificações físicas, químicas e biológicas podem interferir de forma direta ou indireta na distribuição e na biodiversidade dos heteropteros aquáticos. 
Atualmente, as alterações antrópicas nos corpos d'água vêm ocasionando mudanças nos fatores bióticos e abióticos, podendo afetar de positiva ou negativamente a diversidade de espécies presentes (Allan, 2004). Ademais, tanto fatores abióticos como ambientais, podem exercer fortes efeitos sobre a fauna, tais como a sazonalidade, a disponibilidade de água, o desmatamento, as queimadas e a balneabilidade. Esses fatores podem influenciar na integridade do ambiente. Considera-se os Heteropteras um bom modelo para avaliar os impactos ambientais, refletindo em todas as fases da sua vida, com relação às alterações ocorridas nos habitats aquáticos (Dias-Silva et al., 2010; Giehl et al., 2014).

No que se refere aos rios e riachos do estado do Maranhão, pode-se comentar que eles vêm sofrendo diversas ações antrópicas, como a perda de cobertura vegetal, as queimadas, os desmatamentos, e o uso de agrotóxicos para fins agropecuários e madeireiros, principalmente no bioma Cerrado, o que interfere na perda da diversidade das espécies presentes (Nugeo, 2016). Não diferindo do Leste Maranhense, necessita-se conhecer a biodiversidade desses rios e riachos, além de um constante monitoramento dos ambientes, com vistas a sua manutenção e preservação dos ecossistemas florestais e hídricos do território maranhense.

Para o Maranhão, os estudos dos Heteroptera ainda são escassos, destacando-se, contudo, os trabalhos de Azevêdo et al., (2012; 2018) sobre levantamento dos gêneros e Franco et al., (2021) com a lista de espécies de Gerromorpha, embasamento esse que se torna necessário para a realização de novos trabalhos nos riachos do Leste Maranhense que envolvem aspectos taxonômicos e ecológicos da subordem Heteroptera associados a alguns fatores físico-químicos e ambientais dessa subordem.

\section{Metodologia}

As coletas foram realizadas nos igarapés situados na mesorregião do leste Maranhense localizados com ênfase no

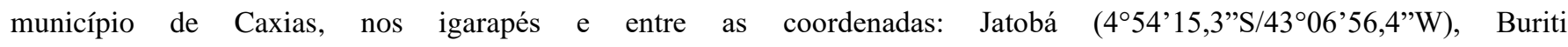

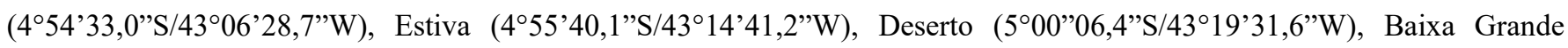

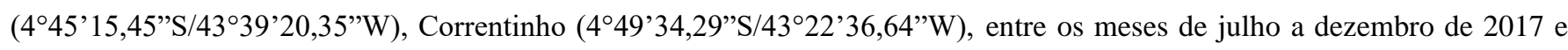
janeiro a junho de 2018.

A região é banhada pelo Rio Itapecuru e possui vários afluentes (Conceição et al., 2010) com rico potencial hídrico. Ademais, é constituída de um clima equatorial semiúmido, com temperatura média anual de $28^{\circ} \mathrm{C}$ e precipitação anual de 1100 a $1800 \mathrm{~mm}$, que atinge o pico na estação chuvosa (dezembro a maio), enquanto a outra metade do ano (junho a novembro) engloba o período de seca (Medeiros, 2015) (Figura 1). 
Figura 1. Mapa (A) Brasil, (B) Estado do Maranhão, círculo perto localização de Caxias. (C) cidade de Caxias, (D) hidrológico do Rio Itapecuru e seus afluentes do município de Caxias - MA. Pontos em vermelho correspondem aos locais amostrados. 1= Baixa Grande; $\mathbf{2}=$ Correntinho; $\mathbf{3}=$ Estiva; $\mathbf{4}=$ Jatobá; $\mathbf{5}=$ Buriti; $\mathbf{6}=$ Deserto.

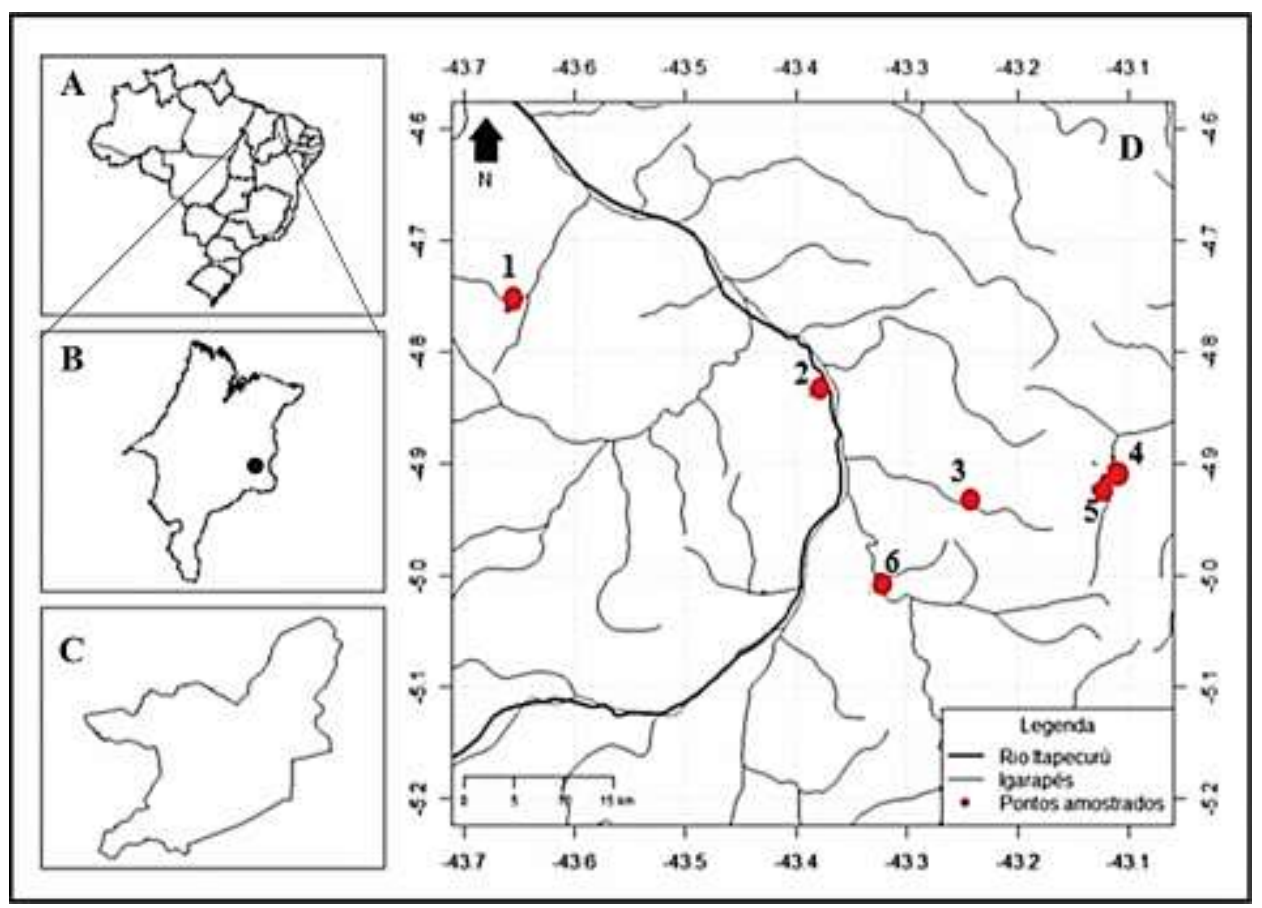

Nota: Os pontos em vermelho correspondem aos locais amostrados. 1= Baixa Grande; $\mathbf{2}=$ Correntinho; 3= Estiva; 4= Jatobá; 5= Buriti; 6 = Deserto. Fonte: Franco (2019).

Em cada riacho, foi delimitada uma seção de 50m dividida em cinco pontos amostrais de 10m, onde os espécimes foram coletados no sentido da jusante para montante para não ocasionar interferência no material coletado (Pereira et al., 2007) (Figura 2). Os Heteroptera foram capturados com rede entomológica em "D” com malha de 1 mm e catação manual, nos substratos folhas, raízes e macrófitas em áreas de correnteza e remanso e sobre a lâmina d'água.

Figura 2. Protocolo de coleta utilizado nos igarapés tributários amostrados para obtenção de imaturos de insetos aquáticos.

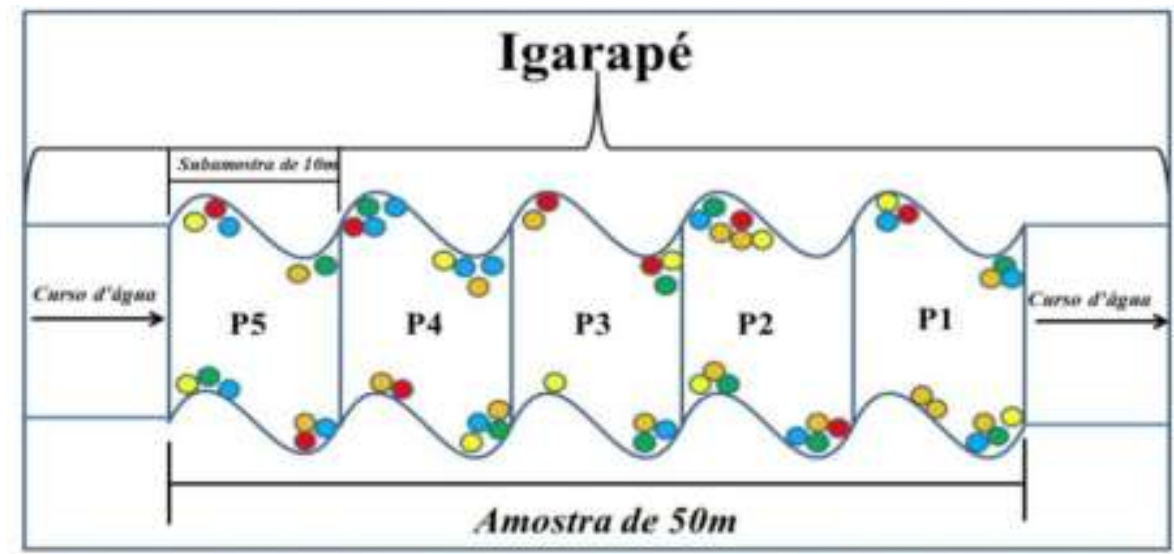

Nota: Os pontos coloridos referem-se à distribuição dos substratos nos riachos. Cores correspondentes: Amarelo (perda); Vermelho (macrófita); Verde (folha); Azul (raiz) e Laranja (tronco). Fonte: Franco (2019).

No local, os substratos foram parcialmente examinados e os espécimes de Heteroptera foram fixados em álcool etílico a $80 \%$. Parte dos substratos foram acondicionados em sacos plásticos para posterior triagem. No Laboratório de Entomologia 
Aquática (LEAq) do CESC/UEMA, o material foi identificado a nível de gênero com a utilização do Estereomicroscópio modelo Stemi DV4 ZEISS e chaves específicas de Pereira et al., (2007) e Ribeiro et al., (2014). Após a identificação, os espécimes foram acondicionados em frascos com álcool a 80\%, etiquetados e depositados no Laboratório de Entomologia Aquática da instituição CESC-UEMA.

Em cada riacho, foram coletadas algumas variáveis físico-químicas e ambientais como temperatura, $\mathrm{pH}$, condutividade elétrica, largura, profundidade, velocidade e vazão (Azevêdo e Hamada, 2006). As coordenadas de cada igarapé foram obtidas por GPS modelo Etrexlegend $\mathrm{H}$ (Garmin); a temperatura, com termômetro digital $\left(0^{\circ}\right.$ a $\left.50^{\circ} \mathrm{C}\right)$; o pH, com pHmetro portátil pH 100 (pHTEK); a condutividade elétrica, com condutivímetro portátil CD - 4301 (Lutron), e a largura foi medida com trena de $50 \mathrm{~m}$.

A vazão e velocidade é estimada pelo método de Craig (1987) que consiste em tomar duas medidas de profundidade com uso de uma régua. Na primeira medida, a régua fica paralela à corrente (D1), e considera-se a profundidade do local; a segunda no mesmo local, vira-se a régua e posicionando-a em ângulo reto em relação à correnteza (D2). Desse modo, é possível calcular a velocidade pela seguinte fórmula: $\mathrm{V}=\sqrt{2}$. $\mathrm{g} . \Delta \mathrm{D}$, em que: $\mathrm{V}=$ velocidade da água, $\mathrm{g}=$ força de gravidade $(19,6 \mathrm{~m} / \mathrm{s} 2), \Delta=$ diferença entre as medidas tomadas com a régua (D2-D1).

\section{Análise Estatística}

A riqueza total de táxons em cada igarapé foi estimada por meio de estimadores de riqueza não paramétricos: Jackknife 1, por avaliar a eficiência do inventário e dos métodos (Magurran, 2004). Além disso, foram construídas curvas de acumulação, para avaliar o quanto o estudo se aproxima da captura de todos os gêneros do local (Hurlbert, 1971). A curva de acumulação de gêneros e os estimadores foram gerados a partir de 100 aleatorizados, utilizando o programa EstimateS 9.1.0 (Colwell, 2019).

A Análise de Coordenadas Principais (PCoA) e a Análise de Redundância (RDA) foram utilizadas para verificar a associação entre as amostras. Outrossim, foi realizada uma matriz de similaridade de Bray-Curtis, em que os dados foram transformados por $\log (x+1)$ (Pavoine et al., 2009). O agrupamento entre gêneros foi expresso por um dendrograma, utilizando-se as médias aritméticas não-ponderadas (UPGMA) da abundância por igarapé. Foi calculado o Coeficiente de Correlação Cofenético (CCC), resultando em um dendrograma com menos distorção do que o coeficiente r de Pearson, em que quanto maior for o r, menor a distorção (Cruz et al., 2011).

A estrutura da comunidade de Heteroptera foi representada pelos eixos da Análise de Coordenadas Principais (PCoA) a fim de atender às premissas de linearidade e ortogonalidade das análises multivariadas (Anderson e Willis, 2003). Quanto ao objetivo, foi o de verificar as diferenças obtidas entre os níveis de especialização e verificar as possíveis relações existentes entre as variáveis respostas obtidas em cada tratamento e possíveis variáveis explicativas. Além disso, foi realizada uma técnica de ordenação conhecida como Análise de Redundância (AR - Redundancy Analysis), A significância das correlações foi obtida pela Correlação de Spearman (Zar, 2010).

Utilizou-se o valor do indicador (Indval) (Dufrêne e Legendre, 1997; De Cáceres et al., 2010) para estimar a especificidade e a fidelidade, bem como avaliar a associação de cada espécie com os tipos de substratos, e potencializá-las para serem utilizadas como indicadores de qualidade ambiental. Os valores de significância foram obtidos pelo Teste de Monte Carlo, utilizando 10.000 réplicas (Dufrêne e Legendre, 1997). Todas as análises foram executadas no programa R (R Core Team, 2020) usando os pacotes Vegan, Stats e Indicspecies.

\section{Resultados e Discussão}

Foram coletados 269 Heteroptera aquáticos e semi-aquáticos em seis igarapés, distribuídos em duas infraordens, sete 
famílias e 17 gêneros (Tabela 1).

Tabela 1. Distribuição de Heteroptera aquáticos coletados em seis igarapés no município de Caxias-MA no Leste Maranhense.

\begin{tabular}{|c|c|c|c|c|c|c|c|c|c|}
\hline Infraordem & Família/Gênero & Sp & $\begin{array}{c}\text { Ig. } \\
\text { BRT }\end{array}$ & $\begin{array}{c}\text { Ig. } \\
\text { JTB }\end{array}$ & $\begin{array}{c}\text { Ig. } \\
\text { ETV }\end{array}$ & $\begin{array}{c}\text { Ig. } \\
\text { DST }\end{array}$ & $\begin{array}{c}\text { Ig. } \\
\text { BAG }\end{array}$ & $\begin{array}{c}\text { Ig. } \\
\text { CRT }\end{array}$ & Total \\
\hline \multirow{11}{*}{ Gerromorpha } & Gerridae & & & & & & & & \\
\hline & Cylindrostethus Fieber, 1861 & Sp1 & 7 & 36 & 0 & 0 & 0 & 0 & 43 \\
\hline & Brachymetra Mayr, 1865 & $\mathrm{Sp} 2$ & 5 & 30 & 1 & 0 & 13 & 0 & 49 \\
\hline & Limnogonus Stål, 1868 & Sp3 & 3 & 1 & 0 & 0 & 0 & 0 & 4 \\
\hline & Trepobates Uhler, 1894 & Sp4 & 1 & 38 & 0 & 0 & 0 & 0 & 39 \\
\hline & Rheumatobates Bergroth, 1892 & Sp5 & 0 & 1 & 0 & 0 & 0 & 0 & 1 \\
\hline & Veliidae & & & & & & & & \\
\hline & Microvelia Westwood, 1834 & Sp6 & 1 & 0 & 0 & 0 & 3 & 0 & 4 \\
\hline & Rhagovelia Mayr, 1865 & Sp7 & 21 & 3 & 0 & 0 & 41 & 0 & 65 \\
\hline & Paravelia Breddin, 1898 & Sp8 & 1 & 1 & 0 & 0 & 1 & 0 & 3 \\
\hline & Stridulivelia Hungerford, 1929 & Sp9 & 0 & 1 & 0 & 0 & 0 & 0 & 1 \\
\hline \multirow{17}{*}{ Nepomorpha } & Belostomatidae & & & & & & & & \\
\hline & Belostoma Latreille, 1807 & Sp10 & 1 & 1 & 1 & 1 & 1 & 0 & 5 \\
\hline & Naucoridae & & & & & & & & \\
\hline & Limnocoris Stål, 1860 & Sp11 & 1 & 0 & 1 & 0 & 1 & 0 & 3 \\
\hline & Ambrysus Stål, 1862 & Sp12 & 1 & 23 & 4 & 8 & 2 & 2 & 40 \\
\hline & Pelocoris Stål, 1876 & Sp13 & 0 & 1 & 0 & 0 & 0 & 5 & 6 \\
\hline & Nepidae & & & & & & & & \\
\hline & Curicta Stål, 1862 & Sp14 & 1 & 0 & 0 & 0 & 0 & 0 & 1 \\
\hline & Ranatra Fabricius, 1790 & Sp15 & 0 & 2 & 0 & 0 & 0 & 0 & 2 \\
\hline & Notonectidae & & & & & & & & \\
\hline & Martarega White, 1879 & Sp16 & 0 & 2 & 0 & 0 & 0 & 0 & 2 \\
\hline & Gelastocoridae & & & & & & & & \\
\hline & Gelastocoris Kirkaldy, 1897 & Sp17 & 0 & 0 & 0 & 0 & $\overline{1}$ & 0 & 1 \\
\hline & Abundância & & 43 & 140 & 7 & 9 & 63 & 7 & 269 \\
\hline & Riqueza & & 11 & 13 & 4 & 2 & 8 & 2 & \\
\hline & Média & & 2,25 & 8,23 & 0,41 & 0,52 & 3,7 & 0,41 & \\
\hline & Das. Pad. & & 5,13 & 13,7 & 1 & 1,94 & 10,1 & 1,27 & \\
\hline
\end{tabular}

Nota: $\mathbf{S p}=$ Espécies enumerada para análises, $\mathbf{I g}=$ igarapé, BRT= Burti, JTB= Jatobá, EST = Estiva, DST= Deserto, BAG= Baixa Grande, CRT= Correntinho. Fonte: Autores.

A curva cumulativa de gêneros, para os seis locais de amostragem iniciou uma curva não assíntota (Figura 3), o que indica a necessidade de novas coletas. O índice Jackknife estimou uma riqueza total de 22,61 \pm as espécies coletadas e indicando que houve diferença significativa entre a curva de riqueza estimada e a curva de riqueza observada.

O Coeficiente de Correlação Cofenético criado (CCC: 0,901) no que se refere à estrutura de comunidades, mostra um conjunto de dados, com corte de 0,55 (50\%) para reduzir a distorção dos dados e se aproximar mais da realidade. Verifica-se a formação de quatro agrupamentos, duas entre comunidades dos riachos de Buriti, Correntinho, Estiva e Deserto e duas amostras de fragmento que ficaram isoladas (Jatobá, Baixar Grande) (Figura 4). 
Figura 3. Curvas de acumulação para Heteroptera contabilizando os indivíduos coletados nos seis igarapés na cidade de Caxias-MA nos anos de 2017 e 2018. Círculo branco esforço amostral e círculo preto estimado Jackknife da análise.

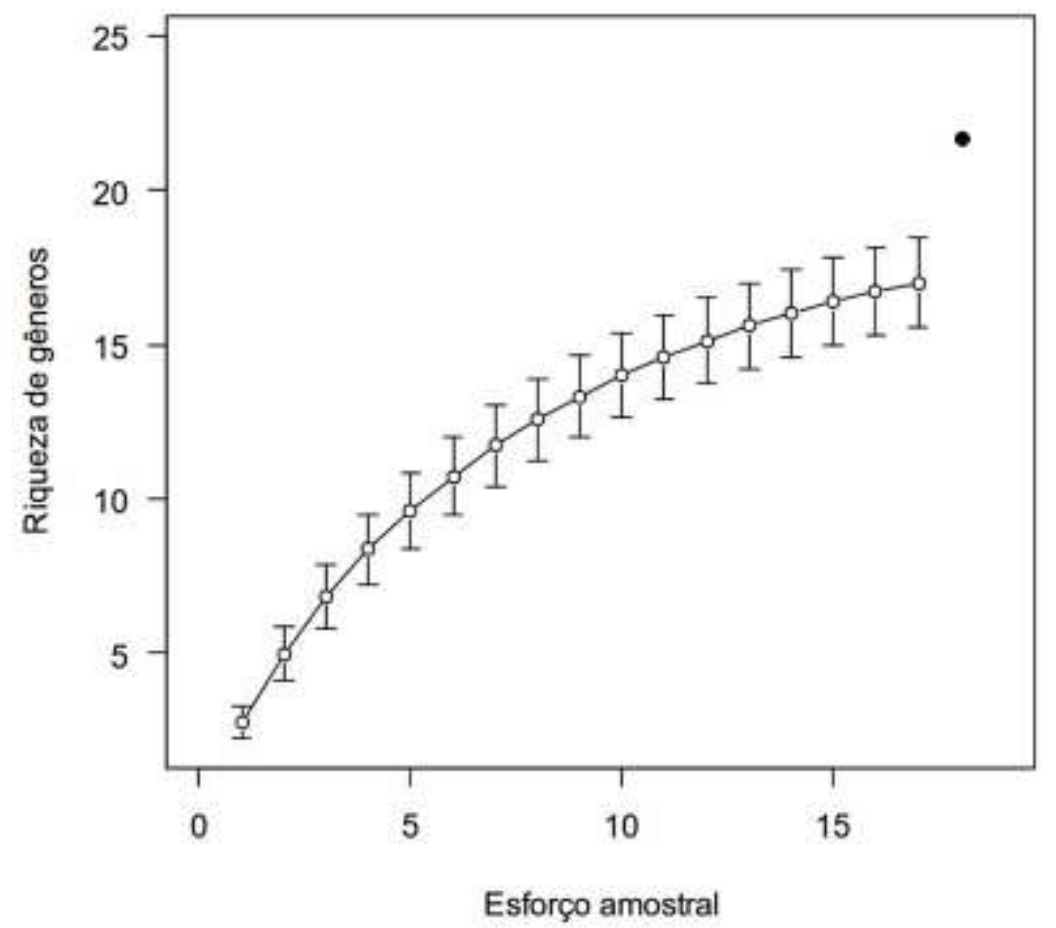

Fonte: Franco (2019).

Figura 4. Diagrama obtido por UPGMA (média não-ponderadas), representando as distâncias medidas entre as amostras comparadas dos seis igarapés na cidade de Caxias-MA nos anos de 2017 e 2018.

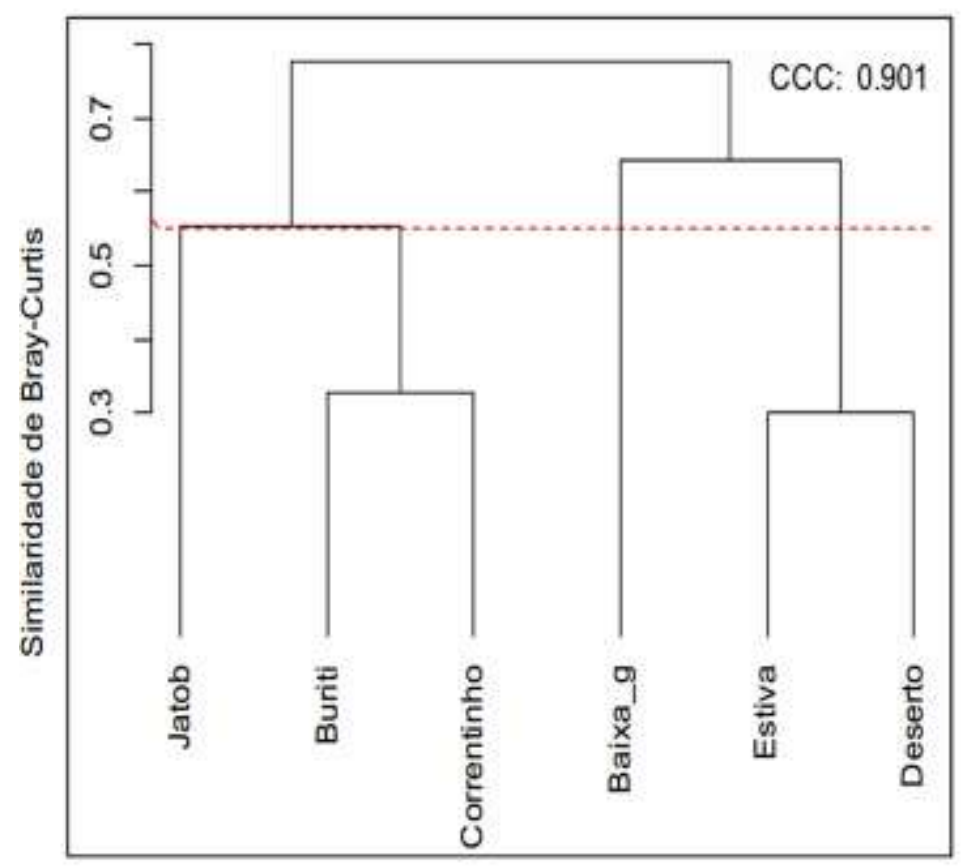

Fonte: Franco (2019).

Na Análise de Coordenadas Principais (PCoA), os dois primeiros eixos explicaram 79,46\% da variação dos dados de espécie. No primeiro eixo, explicou uma variação de 56,22\% e no segundo eixo, explicou 23,24\% da variação dos dados. No 
total, 17 gêneros foram registrados, sendo que os gêneros sp4, sp5, sp9, sp15 e sp16 (Tabela 3) foram exclusivas com o igarapé Jatobá e os gêneros sp17, sp6, sp7 foram fortemente correlacionados ao igarapé Correntinho. Os gêneros sp13 e sp14 foram correlacionados aos riachos Baixa Grande e Buriti, e os riachos Estiva e Deserto não apresentaram correlação aos gêneros encontrados (Figura 5).

Em se tratando da Análise de Redundância (RDA), para as variáveis ambientais, os eixos I e II explicam 43,15\% da variação total dos dados; desses, 29,71\% são explicados pelo eixo I e 13,44 \% pelo eixo II. A variável Vaz.m ${ }^{3}$.s obteve forte correlação com o riacho Baixa Grande; as variáveis Prof/cm e Vel.cm.s se correlacionaram com a espécie sp13; o riacho Jatobá teve forte convergência com os gêneros sp1, sp3, sp4, sp5, sp6, sp8, sp9, sp14, sp15, sp16 e sp17 (Tabela 1) e o riacho Buriti e Correntinho tiveram relação com a espécie sp7, (Figura 6). Os autovalores baseados nos parâmetros ambientais para a RDA nos eixos I e II, as correlações foram significativas para as variáveis: Larg/m (0,78694). Para o eixo I e para o eixo II a variável temp $(0,72091)$ contribuiu para a parte positiva (Tabela 2$)$.

Figura 5. A Análise de Coordenadas Principais(PCoA) com os gêneros relacionados aos igarapés.

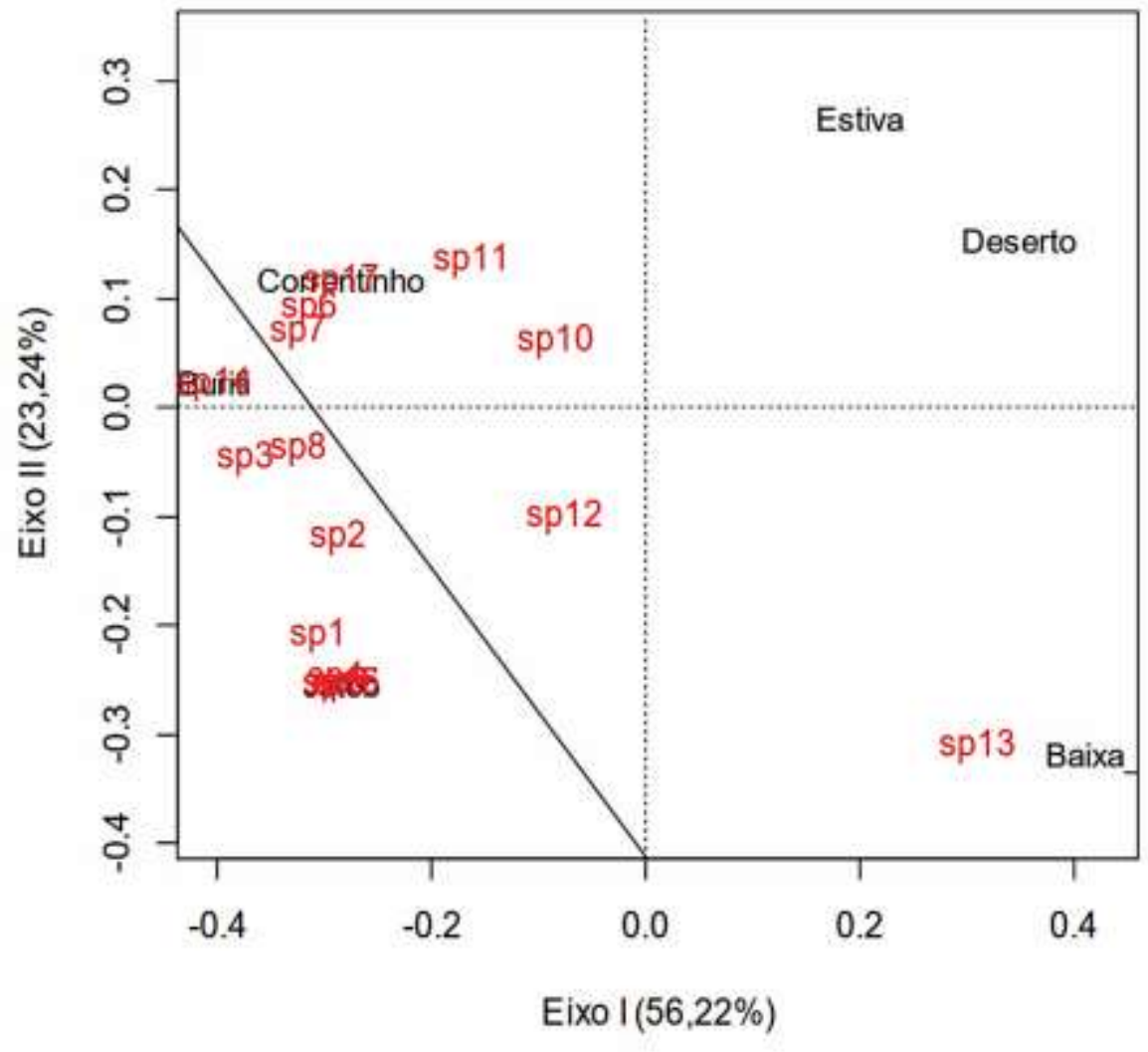

Fonte: Franco (2019). 
Figura 6. Análise de Redundância (RDA) com as variáveis ambientais, correlacionado com os igarapés e gêneros.

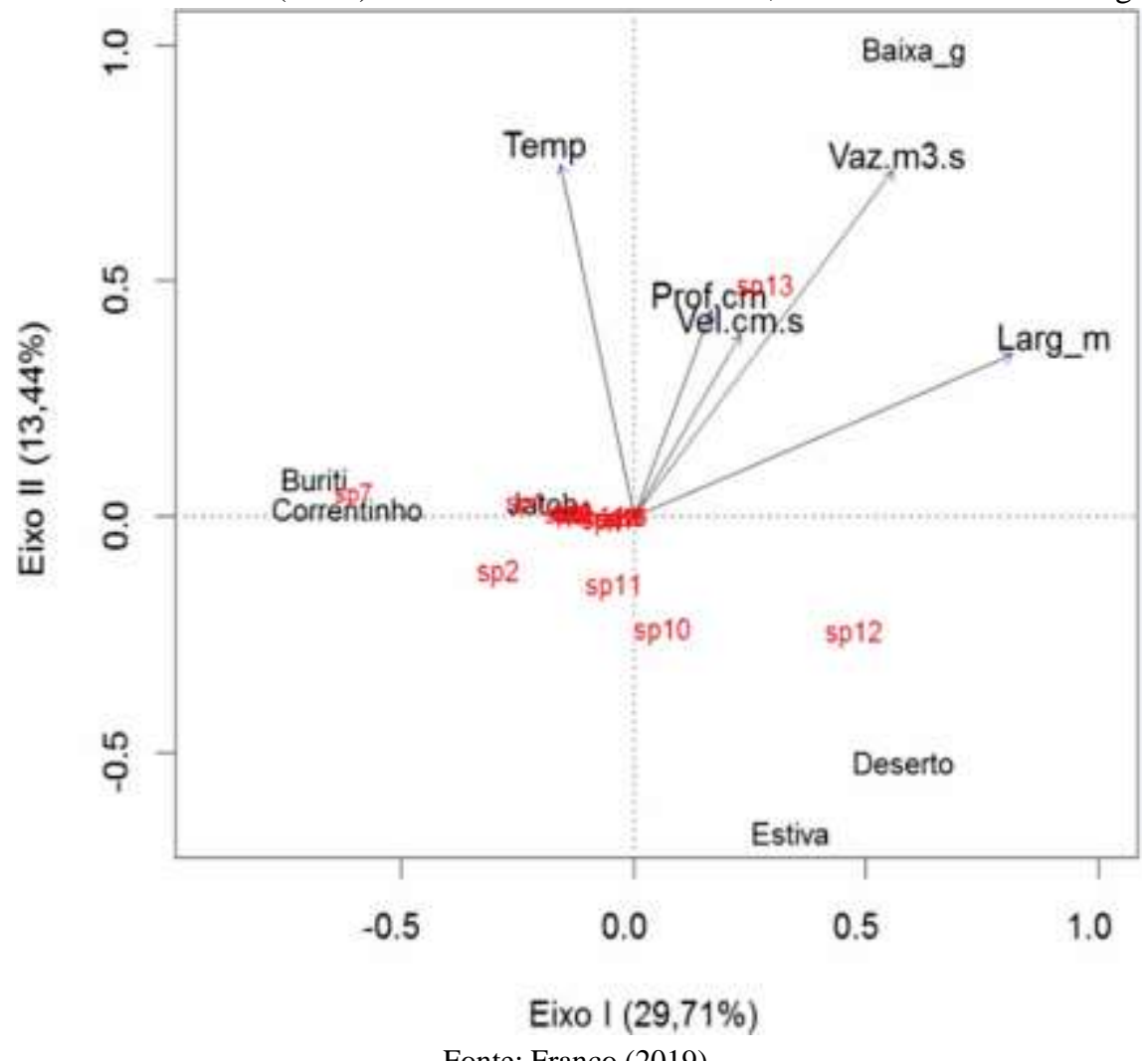

Fonte: Franco (2019).

Tabela 2. Intersecção das correlações variáveis explicativas com as dimensões da RDA.

\begin{tabular}{ccc}
\hline & RDA1 & RDA2 \\
\hline Larg/m & $\mathbf{0 . 7 8 6 9 4}$ & 0.32807 \\
\hline Prof $/ \mathrm{cm}$ & 0.16015 & 0.42288 \\
\hline Vel/cm. s & 0.2195 & 0.37241 \\
\hline Vaz $/ \mathrm{m}^{3} . \mathrm{s}$ & 0.53812 & 0.70725 \\
\hline Temp & -0.1512 & $\mathbf{0 . 7 2 0 9 1}$ \\
\hline
\end{tabular}

Fonte: Franco (2019). 
Tabela 3. Análise de Gêneros Indicadoras (IndVal) de Heteroptera em substrato coletados em riachos, no município de Caxias, Maranhão, Brasil.

\begin{tabular}{lcccccccc}
\hline Gêneros /Código & Folha & Lâmina & Pedra & Raiz & Tronco & Index & stat & p.value \\
\hline Cylindrostethus $(\mathrm{sp} 1)$ & 0 & 1 & 0 & 0 & 0 & 2 & 0.70711 & 0.17 \\
\hline Brachymetra $(\mathrm{sp} 2)$ & $\mathbf{0}$ & $\mathbf{1}$ & $\mathbf{0}$ & $\mathbf{0}$ & $\mathbf{0}$ & $\mathbf{2}$ & $\mathbf{0 . 9 9 3 1 3}$ & $\mathbf{0 . 0 0 5}$ \\
\hline Limnogonus $(\mathrm{sp} 3)$ & 0 & 1 & 0 & 0 & 0 & 2 & 0.70711 & 0.195 \\
\hline Trepobates $(\mathrm{sp} 4)$ & 0 & 1 & 0 & 0 & 0 & 2 & 0.70711 & 0.125 \\
\hline Rheumatobates $(\mathrm{sp5})$ & 0 & 1 & 0 & 0 & 0 & 2 & 0.5 & 0.38 \\
\hline Microvelia $(\mathrm{sp} 6)$ & 0 & 1 & 0 & 0 & 0 & 2 & 0.70711 & 0.205 \\
\hline Rhagovelia $(\mathrm{sp} 7)$ & 0 & 1 & 0 & 0 & 0 & 2 & 0.80724 & 0.175 \\
\hline Paravelia $(\mathrm{sp} 8)$ & 0 & 1 & 0 & 0 & 0 & 2 & 0.61237 & 0.375 \\
\hline Stridulivelia $(\mathrm{sp} 9)$ & 0 & 1 & 0 & 0 & 0 & 2 & 0.5 & 0.38 \\
\hline Belostoma $(\mathrm{sp} 10)$ & 1 & 1 & 0 & 1 & 0 & 17 & 0.57735 & 0.895 \\
\hline Limnocoris $(\mathrm{sp} 11)$ & 0 & 0 & 0 & 1 & 0 & 4 & 0.53137 & 0.605 \\
\hline Ambrysus $(\mathrm{sp} 12)$ & 1 & 0 & 1 & 1 & 1 & 29 & 0.80178 & 0.42 \\
\hline Pelocoris $(\mathrm{sp} 13)$ & 1 & 1 & 0 & 1 & 0 & 17 & 0.44721 & 1 \\
\hline Curictra $(\mathrm{sp} 14)$ & 0 & 0 & 0 & 1 & 0 & 4 & 0.44721 & 0.73 \\
\hline Ranatra $(\mathrm{sp} 15)$ & 1 & 1 & 0 & 0 & 0 & 6 & 0.44721 & 0.82 \\
\hline Martarega $(\mathrm{sp} 16)$ & 0 & 1 & 0 & 0 & 0 & 2 & 0.5 & 0.38 \\
\hline Gelastocoris $(\mathrm{sp} 17)$ & 0 & 0 & 0 & 0 & 1 & 5 & 0.70711 & 0.155 \\
\hline
\end{tabular}

Nota: os valores de $p$ em negrito são estatisticamente significativos $(p<0,05)$. Fonte: Autores.

No estudo dos parâmetros físico-químico dos riachos obteve-se a temperatura média de $26,9^{\circ} \mathrm{C}, \mathrm{o} \mathrm{pH}$ de 6,5 ; a condutividade com média $33,6 \mu \mathrm{s} / \mathrm{cm}$, o oxigênio dissolvido com média de 16,5mg/l, largura média de 4m, profundidade média de $0,88 \mathrm{~cm}$, velocidade média de $0,40 \mathrm{~cm} / \mathrm{s}$, vazão média de $1,55 \mathrm{~m} 3 / \mathrm{s}$. Quanto ao tipo de leito e à largura do dossel, os riachos Jatobá e Estiva possuem o dossel fechado e o leito arenoso; os riachos Buriti, Correntinho, Deserto e Baixa Grande, o dossel parcialmente aberto e o leito arenoso.

Segundo Bachmann (1998); Hamada e Ferreira-Keppler (2012), a diversidade dos Heteroptera se deve à capacidade de dispersão que eles possuem, por poder viver e colonizar diversos ambientes aquáticos, como rios, igarapés, lagos de altitude ou salinos, nascentes, poças de água, marinhos, lâmina d'água planctônica ou associada a plantas (ex: fitotelmatas), troncos, raízes e folhas submersas. Isso lhes confere alta capacidade de dispersão e lhes tornam pioneiros na colonização de corpos d’água. Podem ainda ser encontrados nas margens úmidas, cavernas e regiões costeiras (Mazzucconi et al., 2009).

Outros fatores que podem levar a sua diversidade e dispersão, são as adaptações que alguns podem apresentar, como em Nepomorpha, a presença de pelos hidrofóbicos, o plastrão, assim como em Gerromorpha que impede a entrada de água no sistema traqueal permitindo assim, o hábito patinador (Mazzucconi et al., 2009).

Em relação à família Gerridae, Schuh e Slater (1995) colocam que a maioria dos membros assim como muitos Veliidae, são ativos na superfície da água apresentando um habitat bidimensional. Comenta-se também, que ambos apresentam pernas muito longas com modificações características que facilitam o movimento por meio da tensão superficial da água (Andersen, 1976), o que ocorreu neste estudo com a família Veliidae.

O gênero correlacionado à lâmina d'água, foi o gênero Brachymetra (Tabela 3), entretanto, Rhagovelia foi o gênero com maior abundância na pesquisa. Esse fato pode estar relacionado ao fato de os gêneros serem considerados altamente generalistas, o que possibilita que eles ocupem os mais diversos ambientes aquáticos. A presença de substratos nesses ambientes, são importantes fatores que influenciam a distribuição e riqueza das espécies de Heteroptera aquáticos e semiaquáticos (Ward, 1992) abrigo, alimento e proteção (Resh e Rosenberg, 1984).

Em se tratando dos fatores físico-químicos, estudos apontam para a integridade de habitat, a cobertura vegetal, altitude, vazão, velocidade da correnteza, $\mathrm{pH}$, Condutividade elétrica, temperatura, oxigênio dissolvido e tipos de substratos. 
Essas são as principais variáveis relacionadas à fauna de heterópteros aquáticos e semi-aquáticos (Taylor, 1996; Merritt e Cummins, 1996; Kikuchi e Uieda, 1998; Goulart et al., 2002; Couceiro et al., 2010; Dias-Silva et al., 2010; Ilie e Olosutean, 2013; Peiró et al., 2013). Ressalta-se também, que modificações físicas, químicas e biológicas podem interferir de forma direta ou indireta na distribuição e na biodiversidade dos heteropteros aquáticos. Na presente pesquisa pode-se observar a correlação entre profundidade, vazão e velocidade.

A vazão pode influenciar a fauna de macroinvertebrados aquáticos de diferentes maneiras, visto que quanto maior a vazão e a velocidade da correnteza, mais direta será oxigenação, o potencial erosivo e a hidráulica. Quanto maior a velocidade da água, maior será o carreamento dos substratos e da biota dos insetos aquáticos presentes (Santos Jr et al., 2007). Para que isso não ocorra, alguns heterópteros aquáticos possuem modificações, como achatamento dorso-ventrais (ex. Limnocoris) ou desenvolvem estreita associação com ambientes de alta velocidade como: os gêneros Brachymetra, Cylindrostethus, Microvelia, Paravelia e Tachygerris ou menor como Ambrysus, Ranatra, Stridulivelia e Tenagobia (Pereira et al., 2007).

No que se refere à Teoria do Rio Contínuo, Vannote et al., (1980), enfatizam que são rios pequenos considerados de cabeceira, possuintes de menor tamanho e com maior capacidade de retenção de matéria orgânica e menor profundidade e velocidade; à medida que aumenta esses dois fatores pode-se reduzir a biodiversidade e a distribuição dos organismos presentes. $\mathrm{O}$ aumento da velocidade tende a diminuir a matéria orgânica particulada grossa e aumentar a matéria orgânica particulada fina devido aos efeitos da fragmentação resultante de processos físicos e biológicos. Deve-se levar em consideração que os riachos coletados nesse estudo são considerados de pequeno porte, portanto de média velocidade, vazão e profundidade.

Segundo Merritt et al., (2008) a temperatura pode alterar as condições ambientais, principalmente se houver a ação humana, em que ela, no meio aquático, tende a controlar e influenciar uma série de parâmetros físico-químicos, como o oxigênio dissolvido e a condutividade. Nisso, o avanço da temperatura diminui a solubilidade de gases, como o oxigênio e aumenta a condutividade para uma mesma concentração iônica (Esteves, 1998).

Aos ambientes aquáticos, vale ressaltar que podem sofrer alterações antropogênicas, tais como: desmatamento e perda da mata ciliar, despejo de matéria orgânica e inorgânica, represamento d'água pela construção de estradas e usinas hidrelétricas dentre outros, além da possibilidade de ocasionar alterações que modificam as condições biológicas, físicas e químicas da água, afetando diretamente a sobrevivência de organismos aquáticos (Hamada e Ferreira-Keppler, 2012). Esses fatores estão ocorrendo na maioria dos riachos coletados atualmente, embora observados que ocorrem de maneira lenta. Com isso há a necessidade de maiores estudos e controle nos corpos d'água para que não ocorra a perda da biodiversidade existente.

\section{Conclusão}

Dentre as maiores riquezas dos gêneros apontados no estudo, relaciona-se a avaliação da heterogeneidade de um ambiente. No que tange ao riacho Jatobá, observou-se por meio das análises, que sua maior riqueza, está atrelada a uma melhor correlação entre maioria das espécies, enquanto que o riacho Baixa Grande teve grande relação com a maioria das variáveis ambientes e apenas relação com a espécie Pelocoris, apesar da grande abundância de Rhagovelia. Em lâmina d'água, o gênero que melhor se correlacionou com esse substrato foi o Brachymetra. Nesse viés, pode-se enfatizar que estudos da riqueza e da ecologia são fundamentais para determinar a sensibilidade dos ecossistemas, o conhecimento dos aspectos ecológicos e taxonômicos que se fazem necessários para a conservação desses gêneros. Fornecendo assim informações para futuros trabalhos.

\section{Agradecimentos}

À Universidade Federal Rural do Rio de Janeiro ao Programa de pós-graduação em Biologia Animal, à Universidade Estadual do Maranhão, a Coordenação de Aperfeiçoamento de Pessoal de Nível Superior- Brasil (CAPES) - Código de 
Financiamento 001 e à Fundação de Amparo à Pesquisa e ao Desenvolvimento Científico e Tecnológico do Maranhão FAPEMA.

\section{Referências}

Allan, J. D. (2004). Landscapes and riverscapes: The influence of land use on stream ecosystems. Annual Review of Ecology, Evolution, and Systematics, 35(2002), 257-284.

Andersen, N. M. (1976). A comparative study of locomotion on the water surface in semiaquatic bugs (Insecta, Hemiptera, Gerromorpha). Videnskabelige Meddelelser Fra Dansk Naturhistorisk Forening, 139, 337-396.

Anderson, M. J., \& Willis, T. J. (2003). Canonical analysis of principal coordinates: A useful method of constrained ordination for ecology. Ecology, 84(2), $511-525$.

Azevêdo, C. A. S., \& Hamada, N. (2006). Description of last-instar larva of Corydalus nubilus Erichson, 1848 (Megaloptera: Corydalidae) and notes on its bionomics. Zootaxa, 68(1177), 57-68.

Azevêdo, C. A. S., Desidério, G. R., \& Lima, G. C. (2012). Entomofauna aquática da Área de Proteção Ambiental Municipal do Inhamum, Caxias, Maranhão, Brasil: conhecimento para sua biodiversidade. In M. C. Barros (Ed.), Biodiversidade na área de Proteção Ambiental Municipal do Inhamum (UEMA., pp. 5787).

Azevêdo, C. A. S., Nascimento, S. S. R., Sousa, D. C., \& Aguiar Neto, M. B. (2018). Adultos aquáticos e semiaquáticos da subordem Heteroptera em Igarapés na área de proteção ambiental do Inhamum, Caxias, Maranhão, Brasil. In R. S. Fonseca \& M. C. Barro (Eds.), Avanços das Ciências Biológicas no Centro de Estudos Superiores de Caxias (CESC), Maranhão (UEMA., pp. 53-74).

Bachmann, A. O. (1998). Heteroptera acuáticos. In J. Morrone \& S. Coscarón (Eds.), Biodiversidad de Artrópodos Argentinos. Una Perspectiva Biotaxonómica (Ediciones., pp. 163-180).

De Cáceres, M., Legendre, P., \& Moretti, M. (2010). Improving indicator species analysis by combining groups of sites. Oikos, 119(10), 1674-1684.

Colwell, R. K. (2019). EstimateS: statistical estimation of species richness and shared species from samples. Current Version EstimateS 9.1.0. Retrieved from http://viceroy.colorado.edu/estimates/

Conceição, G. M. da, Ruggieri, A. C., \& Magalhães, E. R. de. (2010). Melastomataceae da Área de Proteção Ambiental Municipal do Inhamum, Caxias, Maranhão. Revista de Biologia e Farmácia, 04(02), 83-88.

Couceiro, S. R. M., Hamada, N., Forsberg, B. R., \& Padovesi-Fonseca, C. (2010). Effects of anthropogenic silt on aquatic macroinvertebrates and abiotic variables in streams in the Brazilian Amazon. Journal of Soils and Sediments, 10(1), 89-103.

Craig, D. A. (1987). Some of what you should know about water or, K.I.S.S.* for hydrodinamics (*keeping it stupidly simple). Journal of the North American Benthological Society, 35, 178-182.

Cruz, C. D., Ferreira, F. M. e Pessoni, L. A. (2011). Biometria aplicada ao estudo da diversidade genética. Visconde do Rio Branco: Suprema.

Dias-Silva, K., Cabette, H. S. R., Juen, L., \& De Marco, P. (2010). The influence of habitat integrity and physical-chemical water variables on the structure of aquatic and semi-aquatic Heteroptera. Zoologia, 27(6), 918-930.

Dufrêne, M., \& Legendre, P. (1997). Species assemblages and indicator species: The need for a flexible asymmetrical approach. Ecological Monographs, 67(3), 345-366.

Esteves, F. A. (1998). Fundamentos de Limnologia. Interciencia/FINEP, Rio de Janeiro.

Giehl, N. F. D. S., Dias-Silva, K., Juen, L., Batista, J. D., \& Cabette, H. S. R. (2014). Taxonomic and numerical resolutions of nepomorpha (Insecta: Heteroptera) in cerrado streams. Plos one, 9(8), 1-7.

Goulart, M., Melo, A. L., \& Callisto, M. (2002). Qual a relação entre variavéis ambientais e a diversidade de heteropteros aquáticos em nascentes de altitudes? Bios.

Hamada, N. e Ferreira-Keppler, R. L. (2012). Guia ilustrado de insetos aquáticos e semiaquáticos da Reserva Florestal Ducke. Manaus: Editora da Universidade Federal do Amazonas.

Hurlbert, S. H. (1971). The nonconcept of species diversity: a critique and alternative parameters. Ecology, 52, 577-585.

Ilie, D. M., \& Olosutean, H. (2013). Aquatic and Semi Aquatic Heteroptera Communities from South-East Transylvanian Small Rivers. Travaux du Muséum National d'Histoire Naturelle “Grigore Antipa," 55(2), 207-216.

Kikuchi, R. M., \& Uieda, V. S. (1998). Composição da comunidade de invertebrados de um ambiente lótico tropical e sua variação espacial e temporal. In J. L. Nessimian \& A. do L. Carvalho (Eds.), Ecologia de Insetos Aquáticos (PPGE-UFRJ., pp. 157-173). Rio de Janeiro, Brasil: Series Oecologia Brasiliensis.

Magurran, A. E. (2004). Measuring biological diversity. Oxford: Blackwell Science.

Mazzucconi, S. A., Ruf, M. L. L., \& Bachmann, A. O. (2009). Hemiptera - Heteroptera: Gerromorpha y Nepomorpha. In Fundación Miguel Lillo (Ed.), Macroinvertebrados bentónicos sudamericanos : sistemática y biología (pp. 167-232). Tucumán. 
Medeiros, R. N. (2015). Visões da natureza. In I. G. Sousa, R. L. de Meneses, \& J. M. Vianna (Eds.), Cartografias invisíveis: saberes e sentires de Caxias (pp. 25-49). Academia Caxiense de Letras. Caxias-MA.

Merritt, R. W., \& Cummins, K. W. (1996). An Introduction to the Aquatic Insects of North America. Journal of the North American Benthological Society, 15(3), 401-403.

Merritt, R. W., Cummins, K. W., \& Berg, M. B. (2008). An Introduction to the aquatic insects of North America. Fouth Edition. Kendall/Hunt Publishing Company (Vol. 45).

Moreira, F. F. F., Barbosa, J. F., Ribeiro, J. R. I., \& Alecrim, V. P. (2011). Checklist and distribution of semiaquatic and aquatic heteroptera (Gerromorpha and Nepomorpha) occurring in Brazil. Zootaxa (Vol. 74).

NUGEO. (2016). Núcleo Geoambiental. Climatologia. https://www.nugeo.uema.br

Pavoine, S., Vallet, J., Dufour, A. B., Gachet, S., \& Daniel, H. (2009). On the challenge of treating various types of variables: Application for improving the measurement of functional diversity. Oikos, 118(3), 391-402.

Peiró, D. F., Saulino, H. H., Gorni, G. R., Corbi, J. J., Vante, A. P., \& Amaral, G. (2013). Insetos Aquáticos Associados a Macrófitas Submersas com Diferentes Complexidades Morfológicas. Revista Brasileira Multidisciplinar, 16(1), 133-144.

Pereira, D. L. V, Melo, A. L. de, \& Hamada, N. (2007). Systematics, morphology and physiology Chaves de Identificação para Famílias e Gêneros de Gerromorpha e Nepomorpha ( Insecta : Heteroptera ) na Amazônia Central, (April), 210-228.

Polhemus, J. T., \& Polhemus, D. A. (2008). Global trends in the description of aquatic and semiaquatic Heteroptera species, 1758-2004. Tijdschrift voor Entomologie, 150(2), 271-288.

R Core Team. (2020). R: A language and environment for statistical computing. R Foundation for Statistical Computing. Vienna, Austria. Retrieved from https://www.r-project.org/

Resh, V. H. e Rosenberg, D. M. (1984). The ecology of aquatic insects. New York: Praeger.

Ribeiro, J. R. I., Moreira, F. F. F.; Barbosa, J. F., Alecrim, V. P. e Rodrigues, H. D. D. (2014). Ordem Hemiptera: Subordem Heteroptera. In: Hamada, N; Nessimian, J. L; Querino, R. B. (Orgs). Insetos Aquáticos na Amazônia brasileira: taxonomia, biologia e ecologia. (Manaus: INPA, 313-333).

Santos Jr, J. E. dos, Strieder, M. N., Fiorentin, G. L., \& Neiss, U. G. (2007). Water velocity and the distribution of larvae and pupae of Chirostilbia pertinax (Kollar) (Diptera, Simuliidae) and associated macroinvertebrates. Revista Brasileira de Entomologia, 51(1), 62-66.

Schuh, R. T., \& Slater, J. A. (1995). True Bugs of the World (Hemiptera: Heteroptera) Classification and Natural History. Cornell University Press, New York, $336 \mathrm{pp}$.

Štys, P., \& Kerzhner, I. (1975). The rank and nomenclature of higher taxa in recent Heteroptera. Acta Entomologica Bohemoslovaca, 72, 65-79.

Taylor, S. J. (1996). Habitat preferences, species assemblages, and resource partitioning by Gerromorpha (Insecta: Heteroptera) in southern Illinois, with a faunal list and keys to species of the state. Ph.D. Dissertation in Zoology, Zoology Department, Southern Illinois University at Carbondale, Carbondale, Illinois, 345 .

Vannote, R. L., Minshall, G. W., Cummins, K. W., Sedell, J. R., \& Cushing, C. E. (1980). The River Continuum Concept. Canadian Journal of Fisheries and Aquatic Sciences, 37(1), 130-137.

Ward, J. V. (1992). Aquatic insect ecology 1. Biology and habitat. John Wiley e Sons, New York.

Zar, J. H. (2010). Biostatistical Analysis (5th ed.). Upper Saddle River: Prentice-Hall/Pearson. 